\title{
Diagnóstico genotípico y fenotípico de tuberculosis ósea y miliar en un paciente positivo para HIV en Bogotá, Colombia
}

\author{
Leonardo F. Jurado', Martha I. Murcia¹, Patricia Hidalgo², \\ John E. Leguizamón¹, Lorena R. González¹ \\ 1 Grupo de Micobacterias, Departamento de Microbiología, Facultad de Medicina, Universidad Nacional de \\ Colombia, Bogotá, D.C., Colombia \\ 2 Unidad de Neumología, Hospital Universitario San Ignacio, Pontificia Universidad Javeriana, Bogotá, D.C., \\ Colombia \\ La tuberculosis se considera la causa más frecuente de muerte producida por un solo agente \\ infeccioso. El diagnóstico de la tuberculosis extrapulmonar no siempre es posible mediante los métodos \\ convencionales debido al lento crecimiento del bacilo y a la naturaleza paucibacilar de las muestras, \\ por lo que es necesario recurrir a las técnicas moleculares. El riesgo de tuberculosis, así como la \\ mortalidad, aumenta en los pacientes con infección por HIV, en quienes el compromiso extrapulmonar \\ es más frecuente. \\ Se describe el caso de un hombre mestizo de 56 años de edad con sospecha de padecer tuberculosis, \\ que asistió a consulta en el Hospital San Ignacio de Bogotá y relató haber tenido dolor en una lesión \\ ulcerada localizada en el tercio distal del antebrazo derecho durante los dos meses anteriores y en \\ quien se confirmó la infección por HIV. \\ El examen histológico de los tejidos óseo y pulmonar demostró la presencia de granulomas múltiples, \\ células gigantes y fibrosis. Tanto los cultivos como la reacción en cadena de la polimerasa en la \\ secuencia de inserción 6110 (insertion sequence, IS6110) fueron positivos. Los aislamientos de \\ Mycobacterium tuberculosis recuperados fueron sensibles a los medicamentos antituberculosos de \\ primera línea.
}

Palabras clave: Mycobacterium tuberculosis, tuberculosis miliar, osteomielitis, ADN, VIH, síndrome de inmunodeficiencia adquirida, reacción en cadena de la polimerasa, Colombia.

doi: http://dx.doi.org/10.7705/biomedica.v35i1.2275

\section{Phenotypic and genotypic diagnosis of bone and miliary tuberculosis in an HIV+ patient in Bogotá, Colombia}

Tuberculosis is the single most frequent cause of death by an infectious agent worldwide. Diagnosis of extra-pulmonary tuberculosis is not always possible through conventional methods, due to the long time required for cultures and the paucibacillary nature of samples; hence the need of rapid molecular methods. HIV infection increases the risk of tuberculosis, and HIV/tuberculosis coinfection is associated with higher mortality.

We describe the case of a 56-year old mestizo male patient suspected of having tuberculosis who consulted the San Ignacio Hospital in Bogotá with a two-month history of a painful ulcerated lesion over the distal third area of the right forearm and in whom HIV coinfection was confirmed.

Bone and pulmonary histological examination evidenced multiple granulomas, giant cells and fibrosis. Cultures and IS6110-PCR from lung and bone tissues were positive for Mycobacterium tuberculosis complex. Mycobacterium tuberculosis isolates were sensitive to first line drugs.

Key words: Mycobacterium tuberculosis; tuberculosis, miliary; osteomyelitis, DNA, HIV, acquired immunodeficiency syndrome, polymerase chain reaction, Colombia.

doi: http://dx.doi.org/10.7705/biomedica.v35i1.2275

\footnotetext{
Contribución de los autores:

Patricia Hidalgo: revisión y análisis de la historia clínica, revisión y aprobación del manuscrito John E. Leguizamón y Lorena R. González: estudios de biología molecular y sensibilidad a antibióticos, revisión y comentario del manuscrito

Martha I. Murcia: actividades de diagnóstico microbiológico y molecular, interpretación de los resultados, revisión y comentario del manuscrito

Leonardo F. Jurado: participación en el diagnóstico molecular, revisión de la historia clínica y la literatura científica pertinente y redacción del manuscrito
} 
La tuberculosis es una de las dolencias humanas más antiguas, pero no por ello es una enfermedad del pasado. Entre la década de 1960 y 1970 la tuberculosis casi había sido eliminada en los países industrializados, pero en los años 90 la enfermedad reapareció, desencadenando una pandemia sin precedentes que llevó a la Organización Mundial de la Salud (OMS) a declarar la emergencia mundial en 1993 (1).

En el 2012 se registraron en el mundo cerca de 8,6 millones de casos nuevos de tuberculosis en todas sus formas, $13 \%$ de ellos entre pacientes positivos para el virus de la inmunodeficiencia humana (HIV). En ese año, 1,4 millones de personas murieron a causa de la enfermedad (160 muertes por hora), de las cuales 430.000 eran positivas para el HIV (2).

En el 2011, Colombia reportó 12.701 casos de tuberculosis en todas sus formas, de los cuales 8.867 fueron de tuberculosis pulmonar y 2.275 de tuberculosis extrapulmonar, en tanto que los casos de infección concomitante con HIV representaron el $11 \%$. Los casos en hombres representaban el $61 \%$, el mayor número de casos se observó entre individuos de 25 a 34 años, aunque los mayores de 65 tuvieron la tasa más alta de enfermedad (Moreno E, Vera N. Colombian tuberculosis situation. International Congress: VI Meeting of the SLAMTB, 2012, Bucaramanga. Med UIS. 2012;25(Supl.):1920). En el mismo año se reportaron en Bogotá cerca de 940 casos de tuberculosis en todas sus formas, la mayoría en personas mayores de 60 años. El porcentaje de infecciones concomitantes fue de $15,7 \%$ : 63,5 \% en adultos jóvenes (27 a 44 años), $83,7 \%$ en hombres y $16,2 \%$ en mujeres (3). En el 2010 se notificaron en el municipio de Armenia 127 casos de tuberculosis, 87 de infección por HIV y 14 de infección concomitante (4).

Entre las razones por la cuales la tuberculosis reapareció pueden mencionarse las siguientes: la pandemia del HIV/sida; la creciente resistencia bacteriana a los fármacos antituberculosos, así como una notable desorganización en los programas de control de la tuberculosis en todo el mundo; el aumento de las migraciones y de la población

\footnotetext{
Correspondencia:

Leonardo F. Jurado, Dirección de Investigación, Fundación Universitaria Navarra, Calle $10 \mathrm{~N}^{\circ} 6-41$, piso 1, Neiva, Huila, Colombia

Teléfono: (578) 8740089

investigacion@uninavarra.edu.co

Recibido: 13/05/14; aceptado: 27/08/14
}

mayor en todo el mundo, y el uso generalizado de medicamentos inmunosupresores para diversas enfermedades y condiciones clínicas $(1,5)$.

La relación entre la tuberculosis y el HIV se evidenció justo después de los primeros reportes de muertes por sida en los años $80(6,7)$. Según reportes de Estados Unidos, durante los últimos años una quinta parte de los casos corresponde a tuberculosis extrapulmonar y hasta un $8 \%$ de los pacientes presentan afectación pulmonar y extrapulmonar simultáneamente (8). Hoy por hoy, el HIV es el factor de riesgo más importante para la reactivación de la tuberculosis latente y de la progresión a enfermedad en los casos de primoinfección o de reinfección con el bacilo tuberculoso (9).Debido a que en los pacientes con HIV la tuberculosis extrapulmonar es más frecuente, el médico siempre debe sospechar su presencia; de hecho, en el ambiente clínico actual se dice: "en todo paciente con HIV se ha de buscar tuberculosis y viceversa" (10), sin olvidar que se trata de la enfermedad que más fácilmente puede ser transmitida por los pacientes con HIV/sida.

\section{Presentación del caso}

Se trató del caso de un hombre mestizo de 56 años de edad proveniente de Armenia, departamento de Quindío, Colombia, que el 5 de septiembre de 2011 presentó dolor, eritema y calor y refirió la aparición de lesiones ulceradas supurativas y dolorosas en el tercio distal del antebrazo derecho, aproximadamente dos meses antes de la consulta; se consideró el diagnóstico de osteomielitis piógena, por lo cual recibió manejo antibiótico empírico con oxacilina y ciprofloxacina administradas ambulatoriamente. La respuesta no fue la esperada, por lo cual el paciente asistió a consulta al Hospital San Ignacio de Bogotá.

Al ingreso, el paciente presentaba tos con expectoración hialina ocasional de un mes de evolución, además de referir episodios febriles.

En el examen físico se encontraron signos de respuesta inflamatoria sistémica con hipotensión (80/43 mm Hg), taquicardia (133 latidos por minuto) y taquipnea (24 respiraciones por minuto), además de compromiso neurólogico con somnolencia, por lo que se consideró la posibilidad de choque séptico; también presentaba adenopatías cervicales anteriores móviles de $3 \mathrm{~cm}$ de diámetro, aproximadamente, no dolorosas y sin signos de inflamación cutánea y placas blanquecinas en lengua y carrillos sugestivas de candidiasis. 
No se detectaron alteraciones en la auscultación cardiopulmonar ni en el examen del abdomen; se encontró lesión acuminada en la región anal; cicatrices violáceas en glúteo derecho, úlcera por presión, grado 1, en zona lumbar y sarcopenia generalizada. En la evaluación neurológica se encontró que el paciente obedecía órdenes, pero evidenciaba desorientación al ser preguntado por su identidad, ubicación y tiempo.

Se procedió a practicar las pruebas de apoyo diagnóstico, las cuales arrojaron los siguientes resultados: en el análisis sanguíneo, el conteo de células blancas fue de 8.300 por $\mathrm{mm}^{3}$,con $87 \%$ de neutrófilos y $8,5 \%$ de linfocitos, $13,3 \mathrm{~g} / \mathrm{dl}$ de hemoglobina, hematocrito de $44 \%, 226.000$ plaquetas por $\mathrm{mm}^{3}$, creatinina sérica de $0,74 \mathrm{mg} / \mathrm{dl}$, nitrógeno ureico en suero de 13,2 mg/dl, sodio de $120 \mathrm{mEq} / \mathrm{l}$, cloro de $87 \mathrm{mEq} / \mathrm{l}$, calcio de $9,7 \mathrm{mg} /$ $\mathrm{dl}$, fósforo de $3,4 \mathrm{mg} / \mathrm{dl}$, potasio de $3,78 \mathrm{mEq} / \mathrm{l}$, magnesio de $2,1 \mathrm{mg} / \mathrm{dl}$, proteína $\mathrm{C}$ reactiva de 4,5 y un conteo de 58 células CD4+.

En la radiografía de tórax se observaron abundantes opacidades micronodulares difusamente distribuidas (patrón miliar), hallazgo que se confirmó tras la realización de una tomografía computarizada de alta resolución en la que, además, se apreciaron las adenopatías cervicales (figuras 1 y 2 ).

Con base en estos hallazgos clínicos y paraclínicos se decidió hospitalizar al paciente y se inició reanimación con líquidos y agentes vasopresores y se le practicaron estudios de extensión.

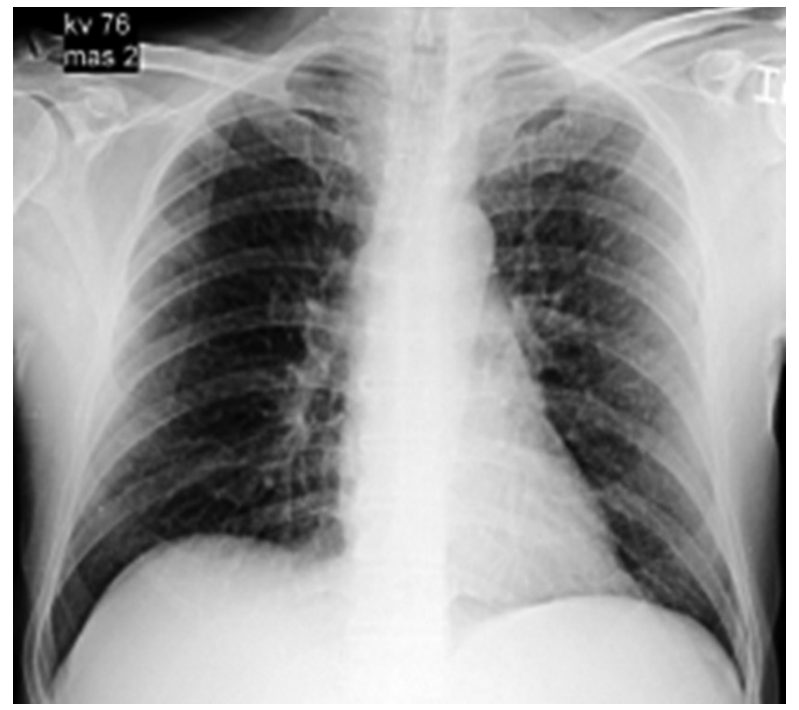

Figura 1. Radiografía simple de tórax en la que se observan abundantes opacidades reticulares y micronodulares difusamente distribuidas (patrón miliar).

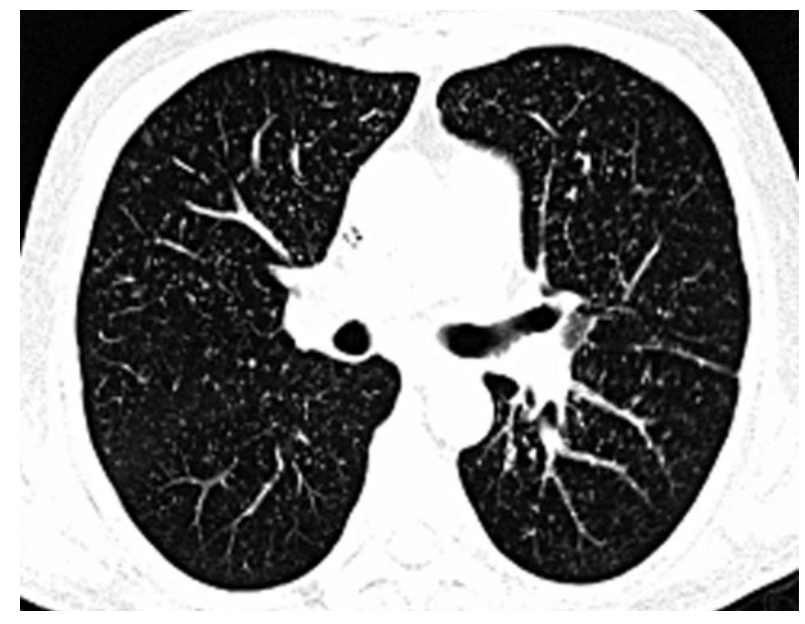

Figura 2. Tomografia de alta resolucion (ventana para pulmón), en la cual se aprecian abundantes lesiones reticulares y micronodulares que comprometen los dos pulmones de manera uniforme (patrón miliar).

Debido a los antibióticos administrados previamente, se consideró la posibilidad de que el agente etiológico de la lesión en el antebrazo fuera Staphylococcus aureus resistente a la meticilina, por lo que se inició el tratamiento con vancomicina.

Con base en la historia clínica, el examen físico y las alteraciones halladas en los exámenes paraclínicos, y previa firma de consentimiento informado por parte del paciente, el 6 de septiembre de 2011 se hizo la prueba ELISA para el HIV con resultado positivo que luego se confirmó mediante análisis con Western blot.

Se practicaron, además, los siguientes exámenes de imágenes: radiografía de mano derecha, en la que se observó osteopenia acentuada y lesión ósea destructiva del carpo (figura 3); tomografía computarizada de cráneo, que evidenció atrofia cortical y ligera dilatación ventricular supratentorial, y ultrasonografía de abdomen total, en la cual se encontró un leve incremento de la ecogenicidad del parénquima hepático, sin lesiones focales evidentes, y una discreta esplenomegalia.

Se hicieron una carpectomía derecha y una lobectomía pulmonar superior izquierda con el fin de tomar muestras para los estudios patológico, microbiológico y molecular; además, se tomaron muestras de líquido cefalorraquídeo para la detección de $M$. tuberculosis con la técnica de la reacción en cadena de la polimerasa (PCR).

En el estudio histopatológico del pulmón se informó abundante infiltrado inflamatorio mononuclear linfocítico, con formación de granulomas 


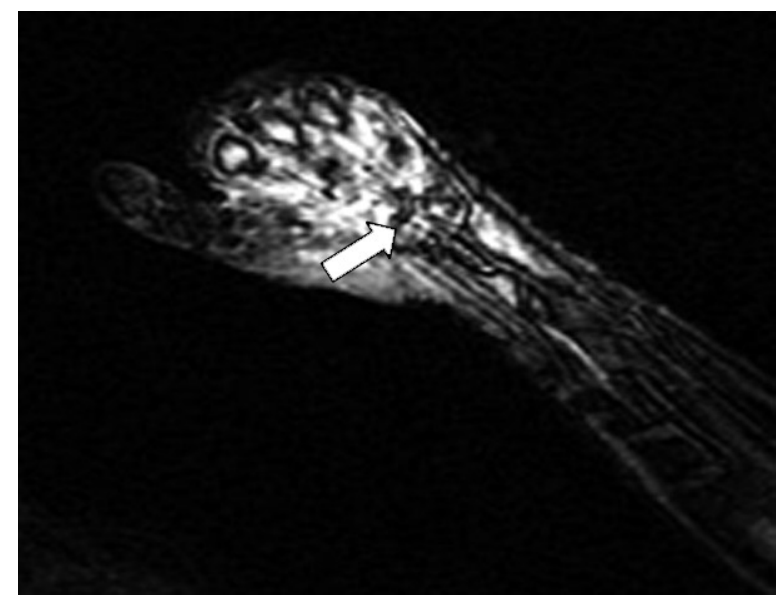

Figura 3. Resonancia magnética nuclear de mano derecha que muestra osteopenia acentuada y lesión ósea destructiva del carpo (flecha).

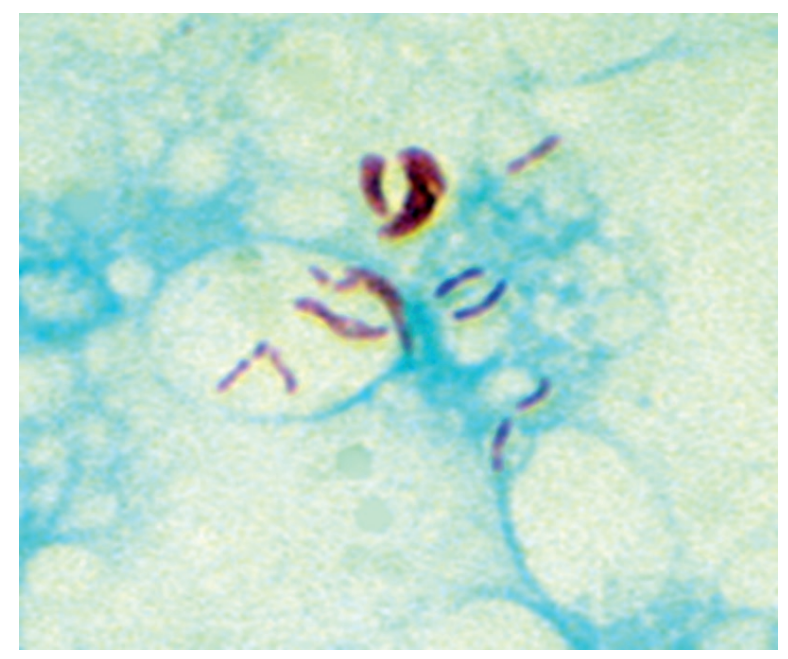

Figura 4. Frotis de biopsia ósea en la que se observan bacilos ácido-alcohol resistentes. Ziehl-Neelsen, 100X.

histiolinfocitarios, necrosis central y células de tipo Langhans; en los estudios de la médula ósea hubo hallazgos similares. Las coloraciones para la detección de bacilos ácido-alcohol resistentes fueron positivas en todas las muestras (figura 4), en tanto que las empleadas para hongos fueron negativas.

Las muestras de tejidos óseo y pulmonar, así como el líquido cefalorraquídeo para el estudio molecular de micobacterias tuberculosas y atípicas (secuencias génicas IS6110 y hsp65), se enviaron al Laboratorio de Micobacterias de la Universidad Nacional de Colombia. Dichos análisis confirmaron la presencia de infección pulmonar, ósea y meníngea por una micobacteria perteneciente al complejo $M$.
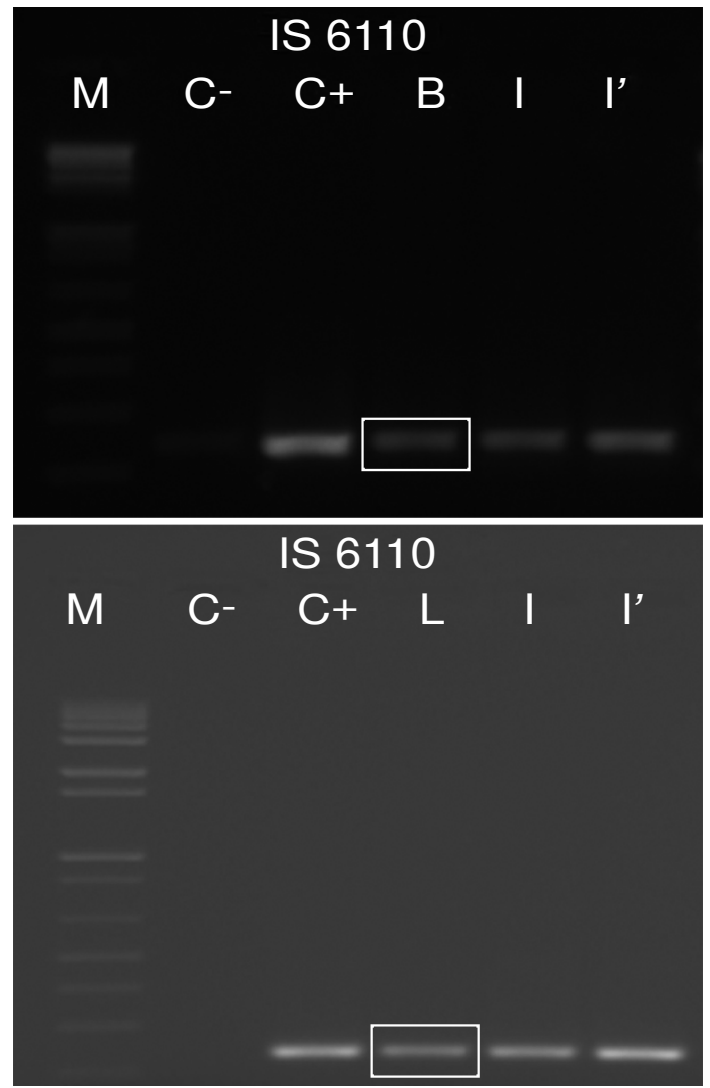

M: marcador de peso molecular (1 Kb plus DNA ladder, Invitrogen); C-: control negativo; $\mathrm{C}+$ : control positivo; $\mathrm{B}$ : espécimen óseo; L: espécimen pulmonar; I: control de inhibidores; I': control de inhibidores diluido 10 veces

Figura 5. Electroforesis de ADN. Los recuadros indican la amplificación del segmento de ADN de 123 pares de bases (blanco de la prueba) en cada espécimen evaluado.

tuberculosis (figura 5). Los cultivos en medio sólido fueron positivos y los aislamientos demostraron sensibilidad a los antifímicos de primera línea.

En respuesta a los hallazgos descritos, se inició el tratamiento antituberculoso con el esquema tetraconjugado (rifampicina, isoniacida, pirazinamida y etambutol); una vez finalizó la primera fase (48 dosis), se comenzó el tratamiento antirretroviral con zidovidina, lamivudina y efavirenz y se continuó con el tratamiento antituberculoso durante 10 meses. La evolución clínica del paciente fue satisfactoria $y$, en la actualidad, continúa asistiendo a controles médicos ambulatorios.

\section{Discusión}

En 1700 John Jacob Manget describió una forma particular de tuberculosis diseminada al asociar la apariencia de las lesiones a pequeños granos de maíz o millo (Zea mays) y acuñó el término de tuberculosis miliar (del latín miliarius - semilla 
molida) para denominarla (11). La tuberculosis miliar es una forma potencialmente mortal de la enfermedad. Las manifestaciones clínicas son inespecíficas y las que se detectan por imágenes diagnósticas son de aparición tardía. En la presentación clínica de la tuberculosis miliar pulmonar son usuales el deterioro acentuado de la función pulmonar y el intercambio gaseoso, situación que se hace evidente en el caso que aquí se reporta (12).

En Colombia, donde la cobertura de la prueba de detección del HIV entre pacientes con tuberculosis es del $50 \%$, la presencia concomitante de tuberculosis y HIV ocurre en el $11 \%$ de los casos (Moreno E, Vera N. Colombian tuberculosis situation. International Congress: VI Meeting of the SLAMTB 2012, Bucaramanga. Med UIS. 2012;25(Supl.): 19-20). En algunos estudios llevados a cabo en diferentes ciudades del país, incluida Armenia, lugar de procedencia del paciente cuyo caso se presenta, las cifras registradas entre 2000 y 2010 difieren entre sí (4). En Bogotá, con una cobertura de la prueba de detección del HIV entre pacientes con tuberculosis del $55,5 \%$, la infección concomitante se detectó en el $16 \%$ de los casos (3); en Armenia, el porcentaje de infección concomitante reportado para el año 2010 fue de 6,8\% (4). En el trabajo en el que se registran estos últimos datos, se señala, además, que el $60 \%$ de los casos se diagnosticaron en instituciones hospitalarias de tercer nivel de complejidad.

La tuberculosis miliar representa entre 1 y $2 \%$ de todos los casos de la enfermedad y hasta el $8 \%$ de los casos de tuberculosis extrapulmonar en individuos inmunocompetentes. La enfermedad es más frecuente en personas inmunodeprimidas (13); no obstante, en otro trabajo publicado en el país se reportaron datos interesantes entre 1998 y 2000: de 89 casos de tuberculosis, $43,8 \%$ eran pulmonares y $56,2 \%$, extrapulmonares; 39 eran de tuberculosis miliar, cuatro de pleural, tres de ganglionar, dos de tuberculosis osteoarticular y dos de tuberculosis meníngea (14). Por otro lado, en un estudio llevado a cabo en Colombia entre 2002 y 2006 en pacientes con tuberculosis e infección por HIV se encontró afectación pulmonar en $37 \%$ de los pacientes, extrapulmonar en $42 \%$ y diseminada en $21 \%$ de los casos (15).

En la era anterior a los antibióticos, la tuberculosis miliar se presentaba predominantemente en lactantes y niños, pero desde los años 70 se ha evidenciado un aumento de la incidencia de esta forma de la enfermedad entre los adultos (16). La pandemia de sida y el uso generalizado de fármacos inmunosupresores ha influido en la incidencia de la tuberculosis miliar. Por otra parte, el efecto de la vacunación con BCG se ha visto reflejado en una reducción sustancial de la tuberculosis miliar y la meningitis tuberculosa en los niños; el creciente uso de la tomografía y de los métodos diagnósticos invasivos han contribuido también al diagnóstico oportuno de la tuberculosis miliar, como se comprobó en el caso que se presenta aquí (17).

El evento crucial en el desarrollo de la tuberculosis miliar es la diseminación linfohematógena masiva de $M$. tuberculosis a partir de un foco generalmente pulmonar, con la subsecuente embolia séptica en diversos órganos (18). La enfermedad puede presentarse inmediatamente después de la infección primaria (tuberculosis primaria progresiva), o a causa de una reactivación favorecida por algún factor inmunosupresor como, por ejemplo, la infección con HIV. La reinfección es frecuente sobre todo en lugares con endemismo elevado (19). Se han reportado casos esporádicos de tuberculosis congénita, en los que la madre padece tuberculosis miliar y la transmisión puede ocurrir por vía placentaria o por aspiración o deglución de líquido amniótico infectante (20).

Aunque en el presente caso solo se documentó el patrón miliar en los pulmones y en la médula ósea, y la PCR en líquido cefalorraquídeo también fue positiva, generalmente todos los órganos con un gran flujo sanguíneo, por ejemplo el bazo, el hígado, los riñones y las glándulas suprarrenales, resultan afectados (18). El análisis macroscópico del órgano afectado suele revelar lesiones redondeadas, pequeñas, de color rojo pálido a gris, y en la histología se evidencia el típico hallazgo de granulomas con centro caseificante. En la infección avanzada por HIV las características cambian notablemente, pues es común encontrar granulomas poco formados, con mínima reacción celular, necrosis seria y bacilos abundantes $(21,22)$. No obstante, en el presente caso el patólogo reportó que el patrón histológico observado tenía las características habituales, aunque sí se detectó la presencia de abundantes bacilos tanto en el tejido pulmonar como en el óseo.

Las manifestaciones clínicas de la tuberculosis miliar son muy variadas e inespecíficas: la enfermedad se puede presentar con fiebre de varias semanas de evolución, anorexia, pérdida de peso, cansancio y tos, todos estos síntomas presentes en el caso que aquí se registra $(12,18)$. 
El diagnóstico preciso y oportuno de la osteomielitis tuberculosa es posible cuando hay un alto grado de sospecha. El diagnóstico se dificulta, entre otras razones, por la falta de familiaridad con el amplio espectro de presentaciones clínicas con las que puede manifestarse la enfermedad tuberculosa $(23,24)$. La frecuencia de la tuberculosis osteoarticular varía, según los reportes, entre el 1 y el $11 \%$ de todos los casos de tuberculosis extrapulmonar, y su forma de presentación más común es la espondilodiscitis (50\%), seguida de la artritis extraaxial de grandes articulaciones (30\%), la osteomielitis (15\%) y la tenosinovitis (3\%) $(25,26)$. Al igual que la mayoría de las demás presentaciones extrapulmonares de la tuberculosis, en especial la miliar, la osteomielitis tuberculosa es secundaria a la diseminación linfohematógena que se produce en la bacteriemia de la infección primaria a partir de un foco pulmonar (25).

La sintomatología de la tuberculosis es muy inespecífica y es por ello que se la conoce como "la gran simuladora". Los pacientes pueden presentar dolor local de aparición insidiosa, hinchazón, disminución de la amplitud del arco de movilidad (lo que se evidenció en el examen físico del paciente del caso que se presenta) y, según la articulación afectada, dificultad para la deambulación. Dependiendo de la extensión de la enfermedad y de la presencia de afección pulmonar concomitante, los pacientes también pueden tener pérdida de peso, diaforesis nocturna, astenia, adinamia e inapetencia, aunque la mayoría de las veces los síntomas locales predominan sobre los sistémicos. En general, la tuberculosis ósea solo se diagnostica cuando ya hay compromiso de la piel y de las estructuras adyacentes, incluidas las articulaciones $(25,26)$, como fue el caso de este paciente.

La tinción de Ziehl-Neelsen para bacilos ácidoalcohol resistentes es la prueba que se usa con mayor frecuencia para el diagnóstico de la tuberculosis pulmonar y extrapulmonar. Los casos extrapulmonares y óseos, en particular, suelen ser paucibacilares, lo que aumenta la dificultad del diagnóstico, incluso cuando la tuberculosis es el diagnóstico diferencial $(21,25,26)$.

Sin embargo, la efectividad de la tinción de ZiehlNeelsen para el diagnóstico de la tuberculosis, incluso de la pulmonar, no es la ideal. Por esta razón, y sobre todo en los casos en los que se sospeche tuberculosis extrapulmonar, es necesario recurrir al cultivo, preferentemente en medio líquido, y a las pruebas moleculares como la PCR, con las cuales, además de proporcionar un diagnóstico rápido, se pueden detectar cargas bacilares de hasta 10 bacilos por $\mathrm{ml}$ de muestra $(25,26)$.

Aunque la prueba de referencia para el diagnóstico de la tuberculosis es el aislamiento del agente etiológico, recientemente se ha reportado que las pruebas más efectivas para el diagnóstico rápido de la tuberculosis osteoarticular son la PCR y el análisis histopatológico del tejido afectado $(21,22,25)$.

Los hallazgos radiológicos incluyen destrucción ósea sin esclerosis, osteopenia yuxtaarticular, secuestro óseo, reacción perióstica, compromiso articular o una combinación de todas estas. A diferencia de la osteomielitis piógena, la tuberculosa no respeta los márgenes articulares y a veces el único hallazgo es una lesión lítica solitaria que puede imitar una neoplasia. El diagnóstico diferencial puede ser muy amplio, y a menudo incluye la osteomielitis piógena crónica, el tumor óseo primario, las metástasis secundarias, las enfermedades granulomatosas por hongos y la artritis inflamatoria, entre otras $(27,28)$.

La presentación clínica de la tuberculosis en personas con infección por HIV, cuyo recuento de células CD4 sea de 500 o más, es similar a la observada en individuos inmunocompetentes. Cuando el conteo de CD4+ es menor de 200 células, como en el caso del paciente que se presenta, las formas graves e inespecíficas de la enfermedad, así como la anergia frente a la tuberculina y la bacteriemia, se hacen más frecuentes, sobre todo en casos de inmunosupresión profunda $(6,7,9,17)$.

Es preciso, entonces, que el médico tenga en mente la tuberculosis como posible explicación de una serie de situaciones clínicas cotidianas y se diga a sí mismo "siempre puede ser tuberculosis".

Una importante condición que debe tenerse en cuenta en los pacientes con diagnóstico de tuberculosis e infección por HIV es la aparición del síndrome de reconstitución inmune una vez se inicia el tratamiento con antirretrovirales, lo que, paradójicamente, conlleva un empeoramiento de los síntomas y las lesiones tuberculosas. Sus manifestaciones incluyen desde casos aislados de fiebre o aumento de las adenopatías y del compromiso pulmonar, aparición de nuevas lesiones, serositis y lesiones cutáneas, hasta compromiso importante del sistema nervioso central, lo que puede llevar a estos pacientes a desarrollar insuficiencia renal aguda o síndrome de dificultad respiratoria aguda. Se ha reportado, igualmente, la aparición 
de lesiones en el sistema nervioso central una vez iniciado el tratamiento para la tuberculosis pulmonar $(9,17,29)$.

El diagnóstico certero de la tuberculosis miliar requiere la obtención de tejidos o fluidos de los órganos presuntamente afectados, así como el estudio histológico respectivo, que en este caso no es el mejor método, y el cultivo, preferentemente en medio líquido (sistema BACTEC-MGIT®), para aislar la bacteria y practicar las pruebas respectivas de sensibilidad $(12,13,17)$.

De los exámenes realizados rutinariamente, la radiografía de tórax puede ser suficiente para un diagnóstico rápido en un contexto clínico específico, pero en lo posible, se deben caracterizar los hallazgos con una tomografía $(18,28)$.

En lo concerniente al tratamiento de los casos en que hay tuberculosis e infección concomitante con HIV, y dadas las interacciones entre algunos fármacos antituberculosos y los antirretrovirales, la OMS por lo general recomienda iniciar el tratamiento antirretroviral una vez se haya finalizado el antifímico, aunque debido al gran riesgo de muerte y complicaciones durante este proceso, sobre todo en pacientes con recuentos de CD4 + menores de 200 , se recomienda iniciar la administración de los antirretrovirales cuanto antes. En el presente caso, dicho tratamiento se inició entre dos semanas y dos meses después de que se había iniciado el tetraconjugado y se comprobara que era bien tolerado $(17-19,30,31)$.

\section{Consideraciones finales}

Como puede verse, después de más de cien años de que el científico alemán Robert Koch describiera el bacilo causante de la tuberculosis, la lucha sin tregua contra la enfermedad continúa e, incluso, se intensifica; para constatar quién va ganando esta batalla basta con revisar los boletines epidemiológicos de todos los países del mundo, sobre todo de las naciones en vías de desarrollo. A la notable reducción en la cobertura de la prueba de detección del HIV en Colombia, se añaden las deficiencias en los sistemas de información epidemiológica, lo cual implica que los datos reportados no muestran la verdadera situación de la infección concomitante y, por ende, que la formulación de las políticas de salud pública no responda a la verdadera dimensión del problema y no se logre el efecto deseado.

Para lograr el control de la tuberculosis y su eventual eliminación es necesario que los países no solo fortalezcan sus sistemas de vigilancia, información y acceso a la salud, sino que haya coordinación entre todos los involucrados con el fin de acabar con la pobreza y la desigualdad. La ciencia necesita más y mejores estímulos. Todos, los estamentos gubernamentales, la sociedad civil, el sector privado y la comunidad afectada por la tuberculosis, son piezas claves en el desarrollo de un programa que garantice el acceso universal a las estrategias de prevención, diagnóstico y tratamiento de todas las formas de la enfermedad.

\section{Conflicto de intereses}

Los autores declaramos no tener ningún tipo de conflicto de intereses.

\section{Financiación}

El desarrollo del presente trabajo no requirió financiación alguna.

\section{Referencias}

1. Lienhardt C, Glaziou P, Uplekar M, Lönnroth K, Getahun H, Raviglione M. Global tuberculosis control: Lessons learnt and future prospects. Nat Rev Microbiol. 2012;10:407-16. http://dx.doi.10.1038/nrmicro2797

2. World Health Organization. Global Tuberculosis Control: WHO report 2013. Geneva: WHO; 2013. Fecha de consulta: 19 de julio de 2014. Disponible en: http://apps.who.int/iris/b itstream/10665/91355/1/9789241564656_eng.pdf.

3. Secretaría Distrital de Salud. Boletín Epidemiológico Distrital de Tuberculosis: semanas epidemiológicas 1 a 52 de 2011, Bogotá. Fecha de consulta: 19 de julio de 2014. Disponible en: http://www.saludcapital.gov.co/sitios/ VigilanciaSaludPublica/Todo\%20ASIS/Boletin\%20TBC\%20 25\%20de\%20mayo.pdf.

4. Arenas NE, Ramírez N, González G, Rubertone S, García A, Gómez-Marín JE, et al. Estado de la coinfección tuberculosis/virus de la inmunodeficiencia humana en el municipio de Armenia (Colombia): experiencia de 10 años. Infectio. 2012;16:140-7.

5. Salgado E, Gómez-Reino J. The risk of tuberculosis in patients treated with TNF antagonist. Expert Rev Clin Immunol. 2011;7:329-40. http://dx.doi.10.1586/eci.11.6

6. Pitchenik A, Fischl M. Disseminated tuberculosis and the acquired immunodeficiency syndrome. Ann Intern Med. 1983;98:112. http://dx.doi.10.7326/0003-4819-98-1-112_2

7. Reichert C, O'Leary T, Levens D, Simrell C, Macher A. Autopsy pathology in the acquired immune deficiency syndrome. Am J Pathol. 1983;112:357-82.

8. Centers for Disease Control and Prevention. CDC WONDER Online Database. OTIS 2009 TB Data, Archive Request TB Elimination, November 2013. Fecha de consulta: $1^{\circ}$ de noviembre de 2013. Disponible en: http:// wonder.cdc.gov/tb-v2009.html.

9. Munawwar A, Singh S. AIDS associated tuberculosis: A catastrophic collision to evade the host immune system. Tuberculosis. 2012;92:384-7. http://dx.doi.10.1016/j.tube. 2012.05.006 
10. Centers for Disease Control and Prevention. Guidelines for Prevention and Treatment of Opportunistic Infections in HIV-infected Adults and Adolescents. MMWR Recomm Rep.2009;58:1-198.

11. Sahn SA, Neff TA. Miliary tuberculosis. Am J Med. 1974;56:494-505.

12. Sharma S, Mohan A, Sharma A, Mitra D. Miliary tuberculosis: New insights into an old disease. Lancet Infect Dis. 2005;5:415-30. http://dx.doi.10.1016/S14733099(05)70163-8

13. Sharma S, Mohan A. Extrapulmonary tuberculosis. Indian J Med Res. 2004;120:316-53.

14. Díaz M, Muñoz S, García L. Tuberculosis en el Hospital Universitario San José, Popayán, 1998-2000. Biomédica. 2004;24(Suppl.):92-101. http://dx.doi.org/10.7705/biomedica. v24iSupp+1.1308

15. Cortés J, Hidalgo P, Rey D, Parra G, Gutiérrez I. Tuberculosis en pacientes con infección por VIH en el Hospital Universitario de San Ignacio, 2002-2006. Infectio. 2007;11:16-22.

16. Jacques J, Solan J. The changing pattern of miliary tuberculosis. Thorax. 1970;25:237-40.

17. Kwan C, Ernst J. HIV and tuberculosis: A deadly human syndemic. Clin Microbiol Rev. 2011;24:351-76. http://dx. doi.10.1128/CMR.00042-10

18. Sharma S, Mohan A, Sharma A. Challenges in the diagnosis \& treatment of miliary tuberculosis. Indian J Med Res. 2012;135:703-30.

19. Sharma S, Mohan A, Kadhiravan T. HIV-TB co-infection: Epidemiology, diagnosis \& management. Indian J Med Res. 2005;121:550-67.

20. Sosa L, Cala L, Mantilla J. Tuberculosis congénita asociada con tuberculosis materna miliar diseminada. Biomédica. 2007; 27:475-82. http://dx.doi.org/10.7705/biomedica.v27i4.168

21. Hunter RL. Pathology of post primary tuberculosis of the lung: An illustrated critical review. Tuberculosis. 2011;91:497509. http://dx.doi.10.1016/j.tube.2011.03.007
22. Jurado LF, Palacios DM, Álvarez J, Baldión M, Campos G. Diagnóstico patológico y molecular de un caso de tuberculosis laríngea primaria en un médico. Biomédica. 2014;34:15-20. http://dx.doi.org/10.7705/biomedica.v34i1. 1624

23. Walker G. Failure of early recognition of skeletal tuberculosis. Brit Med J. 1968;1:682-3.

24. Mannepalli S, Mitchell-Samon L, Guzmán N, Relan M, McCarter Y. Mycobacterium tuberculosis osteomyelitis in a patient with human immunodeficiency virus/acquired immunodeficiency syndrome (HIV/AIDS): A case report. Cases J. 2010;3:67. http://dx.doi.10.1186/1757-1626-3-67

25. Gardam M, Lim S. Mycobacterial osteomyelitis and arthritis. Infect Dis Clin North Am. 2005:4;819-30. http://dx. doi.10.1016/j.idc.2005.07.008

26. Vardhan V, Yanamandra U. Diagnosis of osteoarticular tuberculosis. Ind J Rheumatol. 2011;6:87-94. http://dx.doi. 10.1016/S0973-3698(11)60038-1

27. Soler R, Rodríguez E, Remuninan C, Santos M. MRI of musculoskeletal extraspinal tuberculosis. J Comput Assist Tomograf. 2001;25:177-83.

28. Palestro C, Love C, Miller T. Imaging of musculoskeletal infections. Best Pract Res Clin Rheumatol. 2006;20:1197218. http://dx.doi.org/10.1016/j.berh.2006.08.009

29. Lizarazo J.Aparición paradójica de tuberculomas encefálicos durante el tratamiento de tuberculosis en pacientes inmunocompetentes. Biomédica. 2004;24(Supl):34-42. http:// dx.doi.org/10.7705/biomedica.v24iSupp+1.1300

30. World Health Organization. Treatment of tuberculosis: Guidelines for national programs. 4th ed. Geneva: World Health Organization; 2009. p. 65-72.

31. World Health Organization. Tuberculosis care with TB-HIV co-management: Integrated management of adolescent and adult illness. Geneva: World Health Organization; 2007. p. 25-34. 